\title{
Implementation and Performance Analysis of Remote Inclination Monitoring System
}

\author{
Koray Gurkan*, Burak Akpinar and Ahmet Anil Dindar
}

\begin{abstract}
This paper presents the design, implementation, and test stages of a low-cost inclinometer system. The main block consists of the MEMS-based two-axis inclinometer sensor, custom-designed isolated data acquisition circuit, Raspberry Pi single board computer with open-source Python-based software, and remote FTP server. The selection of hardware components, and the algorithm of software are given in detail. Results of system verification, noise characterization, temperature dependency measurements, and long-term test on a high-rise building have revealed that the inclinometer system is suitable for structural health monitoring applications.
\end{abstract}

Index Terms-Analog-digital conversion, Data Acquisition, MEM/NEMS, Sensors: Inertial, Time-Domain Measurements.

\section{INTRODUCTION}

$\mathrm{S}_{\mathrm{p}}^{\mathrm{r}}$ TRUCTURAL HEALTH monitoring (SHM) systems aim to $\checkmark$ provide a basis for the requirement by installing the sensors on the engineering structures and processing data acquired from the sensors. Smart cities with digital twins also requires the integration of sensing across diverse infrastructure systems. With rapid development in the sensor technology, the monitoring methodologies have evolved from simple to more complex but complimentary measurement techniques. Following the construction phase, tall buildings, bridges, dams are economically important engineering structures and those structures should not be subject to any critical condition during their service life unless a strong excitation (earthquake, blast, flood etc.) or boundary condition change (relative ground settlement) occurs.

KORAY GURKAN, is with Department of Electrical and Electronics Engineering, Istanbul University-Cerrahpasa, Istanbul, Turkey, (e-mail: kgurkan@istanbul.edu.tr).

iD https://orcid.org/0000-0003-2283-8173

*Corresponding Author.

BURAK AKPINAR, is with Department of Geomatics Engineering, Yildiz Technical University, Istanbul, Turkey, (e-mail: bakpinar@yildiz.edu.tr ).

iD https://orcid.org/0000-0002-3076-1578

AHMET ANIL DINDAR, is with Department of Civil Engineering, Gebze Technical University, Kocaeli, Turkey, (e-mail: $\underline{\text { adindar@gtu.edu.tr }) .}$

iD https://orcid.org/0000-0003-3168-8322

Manuscript received May 4, 2020; accepted September 18, 2021. DOI: $10.17694 /$ bajece. 731890
The long-period systems are generally tall structures in which the lateral stability is provided by the vertical load carrying members. Inherently, the tall structures are vulnerable to bend over the vertical axis resulting from second order effects on the structural members. In addition, the structures may slowly lean over to the direction where the soil resistance is poor. This pseudo-static movement causes an increase in the inclination of the building over time, and increasing lateral displacement or inter-story drift occurs towards the upper floors of the building respect to ground floor. As effect of this inter-story drift, local cracks may occur in reinforcement and bearing elements. If a certain inclination limit is exceeded, the building may collapse and this is undesirable [1]. Hence, inclinometers purposed for tilt measurements have attracted considerable attention for structural health monitoring.

General, an inclinometer measures the responses generated by pendulum behavior caused by the earth's gravity. Pendulum type can be liquid, solid mass, or a gas, while measurement method is capacitive, inductive, resistive, magnetic or optic [2]-[6]. SHM concept requires monitoring every tall building, and thus, an optimization between cost and precision is essential. Inclinometers for geodetic applications (i.e., landslide monitoring) have the highest precision, but not suitable for SHM applications as they have a very narrow measurement range and high cost. As micro-electronic mechanical system (MEMS) technology has matured, MEMSbased inclinometer chips have been developed with the advantage of low-cost, small size-factor, high sensitivity, low power consumption, and suitable for mass production. Today, commercial inclinometers using MEMS-based chips employ a method for calculating the tilt based on the difference between the static acceleration and the acceleration due to gravity, using a MEMS type accelerometer.

Behind this commercial products, researchers continue to develop custom inclinometer systems by using these MEMSbased chips to perform novel SHM systems and algorithms. Ha et al. propose a wireless inclinometer sensor node for SHM. The inclinometer used in this study is an Murata SCA103T chip [7]. Huang et al. give detailed performance explanation for a series of MEMS inclinometer prototypes (TJ-UWIS) based on Murata chips developed by the Tongji University [8]. Zhao et al. present high-resolution tilt angle measurement system based on differential amplifier and two SCA103T-D04 single-axis MEMS-based inclinometer chips [9]. Hou et al. present an optimal layout of MEMS 
inclinometers to improve the accuracy of monitoring interstory drift in building structures via rotation measurements using SCA-100T inclinometers [10]. Ando et al. present low cost approach to investigate the seismic response of buildings. The proposed architecture is based on two SCA61T-FAHH1G inclinometers and one triaxial accelerometer [11].

In the above studies, the inclinometer systems generally contain unique electronic hardware with commercial data logging software. Our motivation is to develop a low-cost inclinometer system to work integrated with other sensor elements in a remote SHM network using open-source hardware and software. Here, the development and performance analysis of an inclinometer system, which is the part of the studies to establish a low-cost structural health monitoring network, is proposed. In our early studies [12], [13], the concept and basic structure of the hybrid SHM system was presented, which includes the Global Navigation Satellite System (GNSS), accelerometer and inclinometer. Paper is organized as follows; first, details of sensor, data acquisition hardware and software algorithm are presented. System parameters such as accuracy, output noise, stability, temperature behavior of inclinometer's electronics and thereby extension of its mechanical structure is tested before SHM application. Finally, results of long-term monitoring of 7-story building is given.

\section{SYSTEM DEVELOPMENT}

Block diagram of the inclinometer system is given in Fig. 2 . System includes two isolated circuit blocks for sensing and data acquisition hardware, a microcomputer for local storage and connection to file-transfer-protocol (FTP) server.

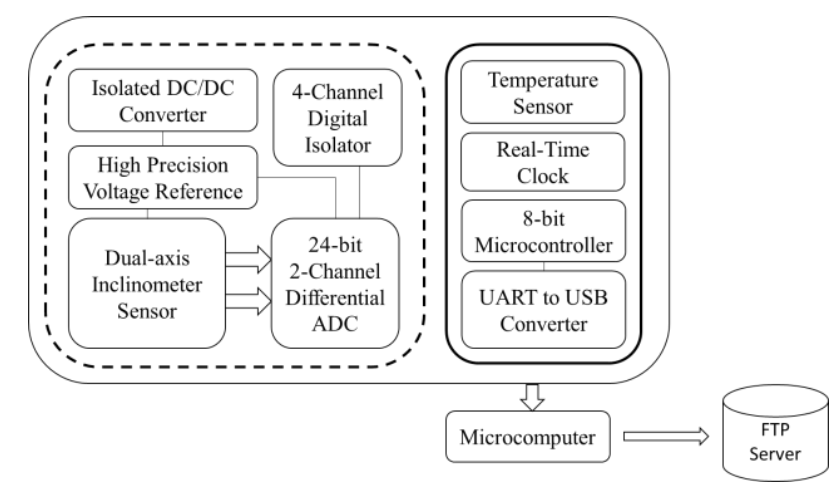

Fig. 2. Block scheme of measurement system

The main part of the system is MEMS based SCA100T-D01 dual-axis inclinometer sensor which has developed by Murata Electronics. As SCA100T has a gravimetric measurement method, it is actually a high gain MEMS accelerometer chip that operates within a certain range. Measurement range of sensor is $\pm 0.5 \mathrm{~g}$ which corresponds to $\pm 30^{\circ}$ inclination. Sensor has ratio metric analog voltage outputs, that is, zero offset point and sensitivity of the sensor are proportional to the supply voltage. In a zero-inclination state, analog output voltage $\left(V_{\text {OUT }}\right)$ of the corresponding axis equals $0.5 V_{D D}$ and varies by the non-zero tilt angle, where $V_{D D}$ is the supply voltage. According to the datasheet this voltage can be transferred to corresponding inclination angle $\alpha$ using following equation;

$$
\alpha=\arcsin \left(\frac{V_{\text {OUT }}-\frac{V_{D D}}{2}}{4}\right)
$$

For nominal value of $V_{D D}=5.0 \mathrm{~V}$ at zero-inclination position $(\alpha=0)$ analog output of any axis will be $2.5 \mathrm{~V}$ each. As seen in (1), if the SCA100T supply voltage $V_{D D}$ fluctuates, the SCA100T output will also vary. To minimize this fluctuation, a voltage reference has to be used as analog supply voltage source which has low noise, high accuracy and temperature stability. For this reason, ADR4550B voltage reference is used which has $5.000 \mathrm{~V}$ output and $\pm 1 \mathrm{mV}$ initial output voltage error and maximum temperature coefficient of $2 \mathrm{ppm} /{ }^{\circ} \mathrm{C}$. ADR4550B has $5.3 \mathrm{~V}$ to $15 \mathrm{~V}$ input voltage range and $10 \mathrm{~mA}$ output current.

For digital conversion of analog data, ADS1232 24-bit analog-to-digital converter (ADC) is used. It has 2-channel differential inputs and delta-sigma topology which is better for low-noise conversion. Previously introduced voltage reference is connected to analog supply voltage and reference voltage input of ADC. When the same reference voltage for both the SCA100T sensor and analog digital converter is used, the error caused by reference voltage variation is automatically compensated. As noise level will be better for lower bandwidth and inclinometer data has generally pseudo-static characteristic, ADC is chosen to be operated at 10 SPS.

To prevent additional external noise, ground loops must be eliminated which requires galvanic isolation of measurement system. Analog and digital power of sensor board and their ground layers has to be also separated as a rule of thumb. Four-channel ADuM5402 digital isolator is used to isolate measurement system from microcontroller board and other peripherals. Analog power of isolated side is supplied separately via TMA $1212 \mathrm{~S}$ isolated dc-to-dc converter which is connected directly input of the precision voltage reference.

Digital output of ADC which converts analog sensor data is read by a low-power 8-bit microcontroller chip ATmega328AU. Ambient temperature is measured by same microcontroller with using AM2302 temperature sensor which has $\pm 0.1^{\circ} \mathrm{C}$ resolution and $\pm 0.5^{\circ} \mathrm{C}$ accuracy. To timestamp all sensor data an extremely accurate real-time clock (DS3231) is used with accuracy of $\pm 3.5 \mathrm{ppm}$ from $-40^{\circ} \mathrm{C}$ to $+85^{\circ} \mathrm{C}$. It has a square wave output and its frequency is selected as $1 \mathrm{~Hz}$ to trigger microcontroller. Date, time information, and scaled sensor data packet transferred at each second period with following format;

\section{$19.09 .2019,16: 20: 04,28.90,0.01957864,0.01397163\langle\mathrm{CR}\rangle\langle\mathrm{LF}\rangle$}

Here, values are date, time, temperature in Celsius, sensor outputs for $\mathrm{x}$ and $\mathrm{y}$ directions in Volts, CR and LF are carriage return and line feed characters, respectively. Total size of transmitted characters is minimum 49 bytes per second for positive values, and maximum 52 bytes per second when sign 
of all data is negative. To transfer data packet to microcomputer, UART protocol is used with 115200 baud rate, 8-bit data size, 1 stop bit, and no parity settings. An UART-to-USB converter is used for easy connection of the system to a microcomputer.

Printed circuit board (PCB) of hardware has been designed with a circular shape which has $50 \mathrm{~mm}$ diameters, and fabricated with PCB prototyping machine (MITS Electronics Eleven Lab) via milling of copper clad laminate. After successful primary tests, PCBs were manufactured with a copper thickness of $35 \mu \mathrm{m}$ and $1.6 \mathrm{~mm}$ thick FR-4 TG130 material (Fig. 3).

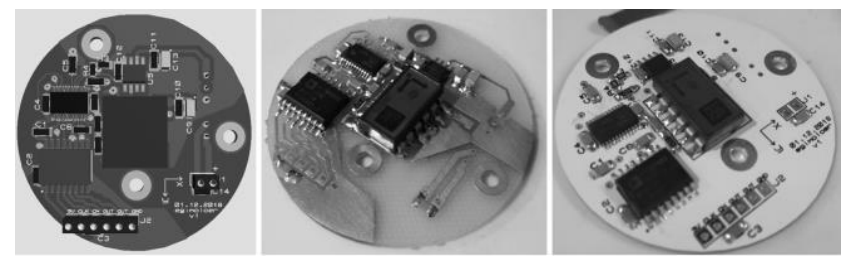

Fig. 3. Designed and fabricated PCBs

As given in Fig. 4 system consists of two isolated PCBs. The top PCB includes inclinometer sensor, ADC, voltage reference and digital isolator. RTC, temperature sensor and microcontroller is located at the bottom PCB.
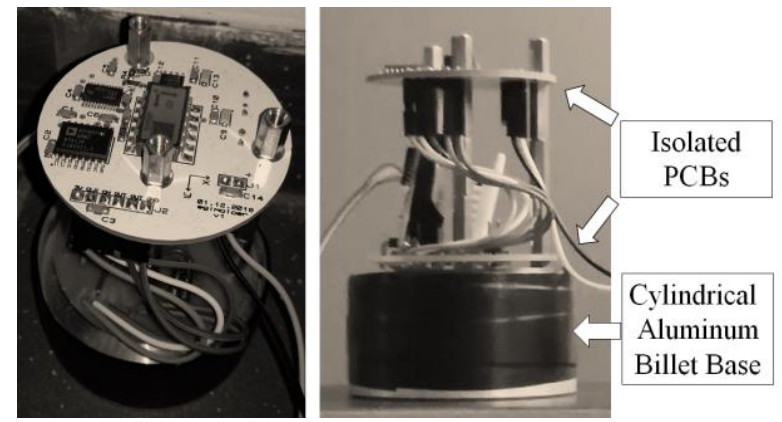

Fig. 4. Top and side view of inclinometer system

Isolated PCBs mechanically coupled with male-female threaded hexagon metal M3 standoffs. The lower base carrying the whole system is made of cylindrical aluminum billet to provide stable operation.

For local and remote storage, an FTP client is generated with Raspberry Pi 3 B model single board computer. Operating system is Debian based Raspbian OS which is the most used Linux for Raspberry microcomputers. Raspberry Pi is connected to the internet via Ethernet connection. Software for inclinometer data recorder and FTP file transfer is developed under Python ${ }^{\circledR}$ (version 3.7.1) open-source environment. Through the development of software, serial connection, file writing, FTP authorization and file transfer connection operations realized by importing serial, os, ftplib and shutil Python libraries.

Software reads incoming data from serial port at each second. After parsing analog voltage outputs, temperature, date and time data, a file is created for writing append mode which named as "INC_Dev_DDMMYYYY_HH00" format and recorded for 1-hour interval. Here, INC indicates it is an inclinometer data file, Dev is three-digit device ID. For example, if software is running on device " 001 " and start recording data at 26.09.2019 13:20:14, opened file name will be INC_001_26092019_1300. After 13:59:59, a new file named INC_001_26092019_1400 is automatically created and sensor data will be written to this file after that, and old file INC_001_26092019_1300 will be send to FTP Server after internet connection checking and FTP authorization. As date information is already located in opened file name, only time, temperature and analog voltage outputs are recorded to the opened file. For 1-hour duration total file size is nearly 141 kBytes, and 3.29 Mbytes for 1-day period. Bill of material and total system cost is given in Table 1 .

TABLE I

BILL OF MATERIAL

\begin{tabular}{llll}
\hline Material & Definition & Manufacturer & $\begin{array}{l}\text { Price } \\
(\$)\end{array}$ \\
\hline SCA100T-D01 & 2-axis MEMS Sensor & Murata Electronics & 35 \\
ADS1232 & 24-bit ADC & Analog Devices & 5 \\
ADuM5402 & 4-channel Digital Isolator & Analog Devices & 12 \\
ADR4550B & Voltage Reference 5V & Analog Devices & 9 \\
DS3231 & Real-Time Clock & Maxim Integrated & 8 \\
AM2302 & $\begin{array}{l}\text { Temperature and } \\
\text { Humidity Sensor }\end{array}$ & Aosong Electronic & 10 \\
ATmega328AU & Microcontroller & Traco Power & 5 \\
R-Pi 3 B Model & Single Board Computer & Microchip & 2 \\
Others & $\begin{array}{l}\text { PCB production, SMD } \\
\text { resistors, capacitors, } \\
\text { mechanical components }\end{array}$ & & 35 \\
TOTAL & & & 15 \\
\hline
\end{tabular}

\section{VERIFICATION AND CHARACTERIZATION}

To verify voltage-to-tilt conversion of inclinometer system, a tailor made angle adjustable inclined plane board platform is build (Fig. 5). A cantilever supported with two metal hinges from one side and a screw with other side. By trimming screw height, inclination changed with $0.5^{\circ}$ per step according to a reference digital level and protractor (INSIZE) which has $0.1^{\circ}$ resolution and $0.2^{\circ}$ accuracy. For each step 60 samples were collected and averaged. The operation was performed for both axes. Reference device has a tare mode which operates by reading the inclination angle when "zero" button is depressed and storing that angle as the zero inclination angle of the device. For each axes, ground floor referenced as zero inclination angle before measurements. 


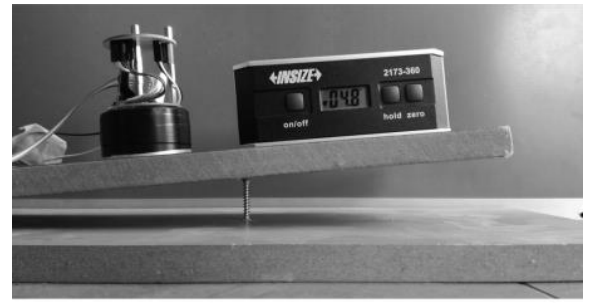

Fig. 5. Tailor made adjustable inclined plane board platform

The measured inclinometer data with respect to reference device readings and fitted curves is shown in Fig 6. The linear fit produced an R-square of 0.997 for both axis indicating an excellent correlation and linearity between the reference device and measured tilt angle.
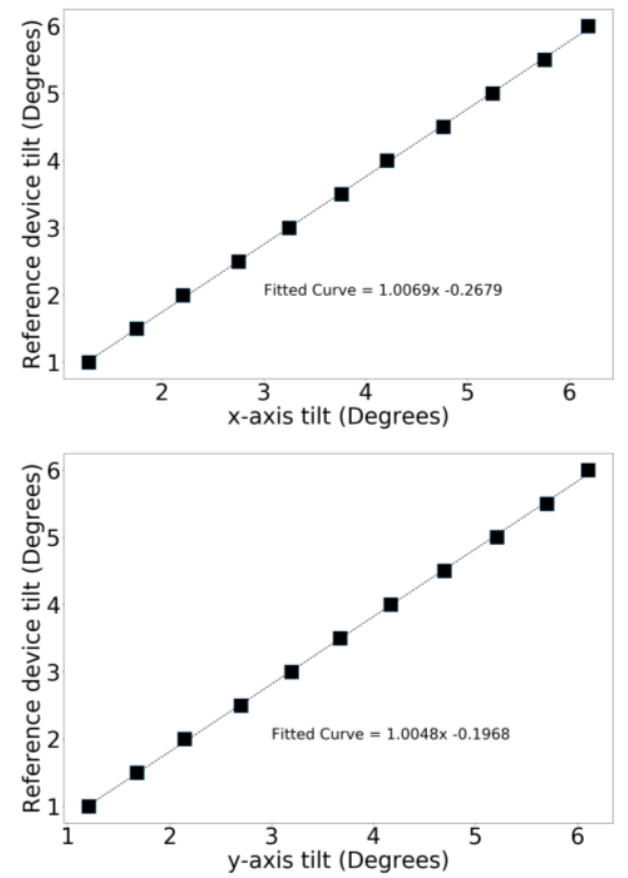

Fig. 6. Reference device readings and fitted curves for verification.

Temperature dependency of the bias, sensitivity, stability, and self-noise of the inclinometer are the most key parameters that directly affect the long-term tilt measurement accuracy. Typical offset and sensitivity temperature dependencies of the SCA100T are presented in datasheet. However, these dependencies are only specific for sensor itself. After processes in system production like PCB manufacturing, soldering, mechanical assembly, etc., temperature dependency of total system has to be measured again to prevent incorrect inclination measurements in environments where dynamic temperature change is high or change of inclination is small.

For achieving temperature characterization of inclinometer system, it has placed inside a PID controlled incubator. First, target temperature has settled to $40^{\circ} \mathrm{C}$ while ambient temperature is $28^{\circ} \mathrm{C}$. After reaching stable target temperature, the new target temperature is settled to $50^{\circ} \mathrm{C}$ and then $60^{\circ} \mathrm{C}$. For each step, total duration of measurement is at least 2 hours to guarantee steady-state data.

Measurement data of temperature dependency test has given in Fig. 7. Here, temperature dependency of each axis is modeled with Levenberg-Marquardt algorithm using Google Colab environment and SCIpy curve fitting package.

The equation of fitted $3^{\text {rd }}$ order polynomial curve for temperature dependency is:

$$
I N C(T)=I N C_{0}+\alpha\left(T-T_{0}\right)+\beta\left(T-T_{0}\right)^{2}+\gamma\left(T-T_{0}\right)^{3}
$$

where $I N C_{0}$ is measured inclination data at temperature $T_{0}$ in ${ }^{\circ} \mathrm{C}$, and $\operatorname{INC}(T)$ is calculated inclination for temperature $T$, respectively. Optimal values of coefficients and asymptotic standard deviation of prediction error of curve fitting process for each axis is given in Table II.
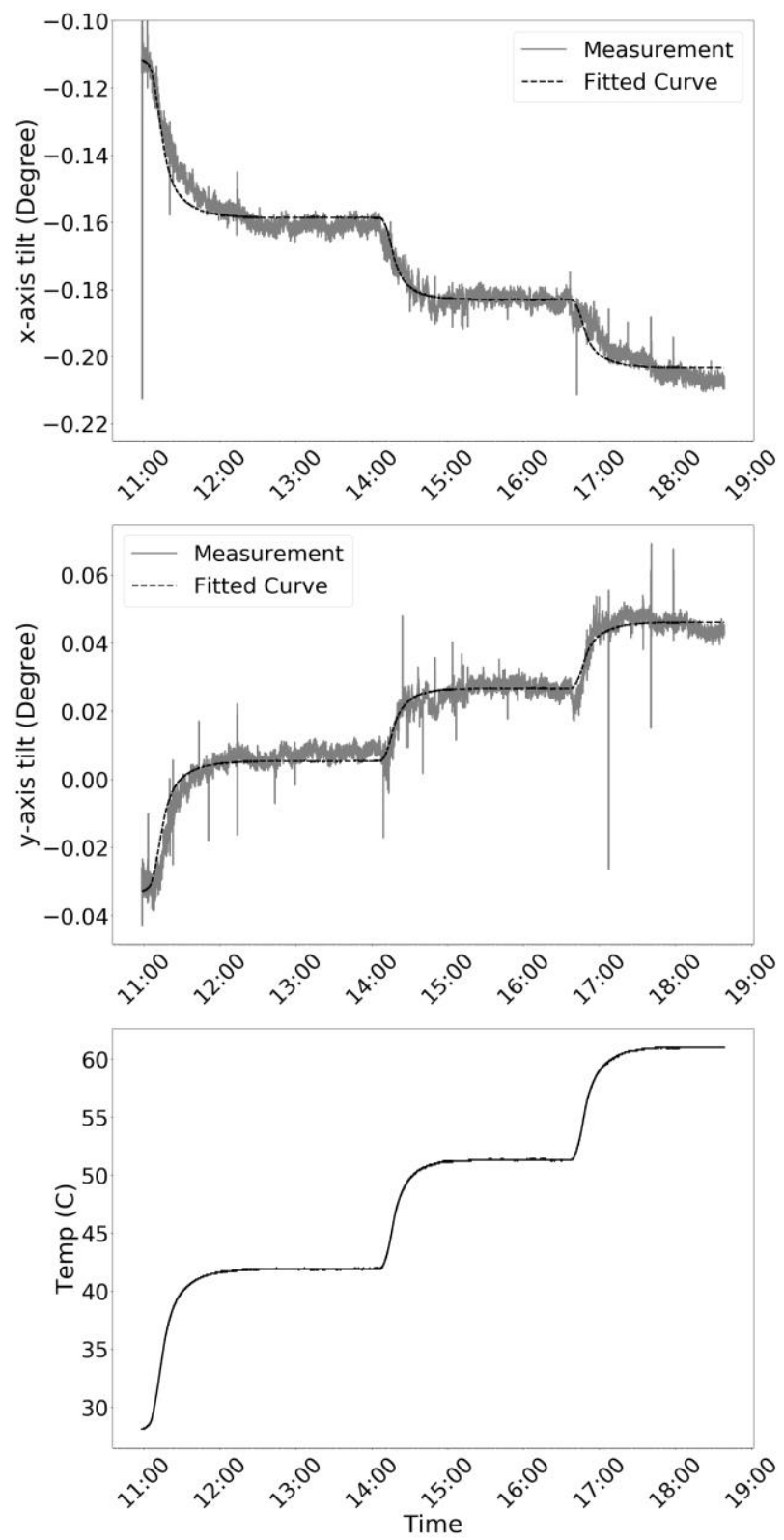

Fig. 7. Inclinometer data and fitted curves respect to variable temperature

For each axis temperature compensated inclination $\left(I N C_{\text {comp }}\right)$ can be found with subtracting calculated $I N C(T)$ value from $I N C_{\text {measured }}$ measured inclination data; 


$$
I N C_{\text {comp }}=I N C_{\text {measured }}-I N C(T)
$$

TABLE II

CURVE FITTING PARAMETERS

\begin{tabular}{ccccc}
\hline \hline \multirow{2}{*}{ Axis } & $\alpha$ & $\beta$ & $\gamma$ & $\sigma_{\varepsilon}$ \\
\hline$x$ & $-3.93 \mathrm{e}-03$ & $4.25 \mathrm{e}-05$ & $-2.27 \mathrm{e}-07$ & $1.17 \mathrm{e}-04$ \\
$y$ & $+3.11 \mathrm{e}-03$ & $-2.85 \mathrm{e}-05$ & $+2.02 \mathrm{e}-07$ & $3.51 \mathrm{e}-04$
\end{tabular}

Short-term standard deviations and long-term Allan deviations are analyzed for each direction to achieve noise characterization and noise level measurement of system. For standard deviation calculation 600 points of inclination data were selected from the region where the temperature change is zero. According to calculations, the short-term standard deviation of inclination in the $\mathrm{x}$ and $\mathrm{y}$ directions are $0.00174^{\circ}$ and $0.00179^{\circ}$, respectively.

Fig. 8 shows the Allan deviation log-log curve for 8-hours of inclination data. After linear curve fitting, slope of Allan deviation curve is determined as -1 for each direction which means that quantization noise is dominant. From this graph corresponding Allan deviation in $\mathrm{x}$ and $\mathrm{y}$ directions at cluster size $\sqrt{3}$ are $0.00166^{\circ}$ and $0.00171^{\circ}$, respectively, which theoretically give quantization noise levels.

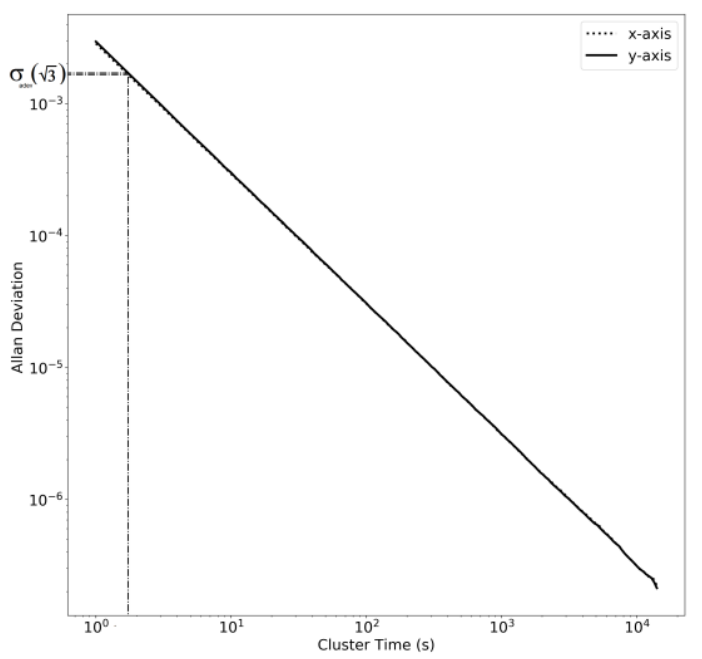

Fig. 8. Allan deviation plots for 8-hours of inclination data

\section{SHM APPLICATION}

Inclinometer system is tested on a 7-story building which is located at Istanbul University-Cerrahpasa Avcilar Campus approximately $15 \mathrm{~km}$ north from the seismogenetic North Anatolian Fault Zone (NAF) in the Marmara Sea region (Fig. 9). The building is laying on friction pile deep foundation due to clayey silty sand lenses levels which prone to soil amplification during an earthquake or swelling and differential settlements [14].

Inclinometer system is installed at 4th floor of building which is 17 meters' height from the ground floor. Sensor data was successfully recorded for 2 months without any data loss, mains failure or connection error.

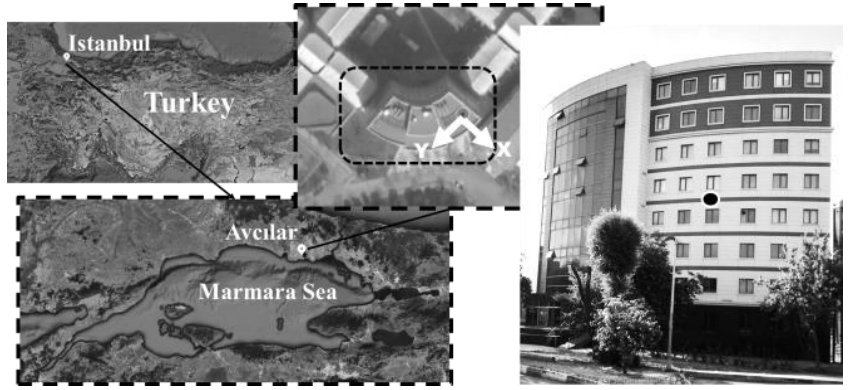

Fig. 9. Satellite view, location and reference directions of test building

Recorded data is analyzed at Google Colab environment using Python Data Analysis (Pandas), SciPy libraries. Inclination data of each axis is filtered using $2^{\text {nd }}$ order Butterworth digital low-pass filter using SciPy filtfilt function. Long-term inclination data compensated with temperature using (2). Filtered, compensated inclination, and temperature data is fitted to a linear curve as a function of time to model pseudo-static inclination and temperature change of building (Fig. 10).
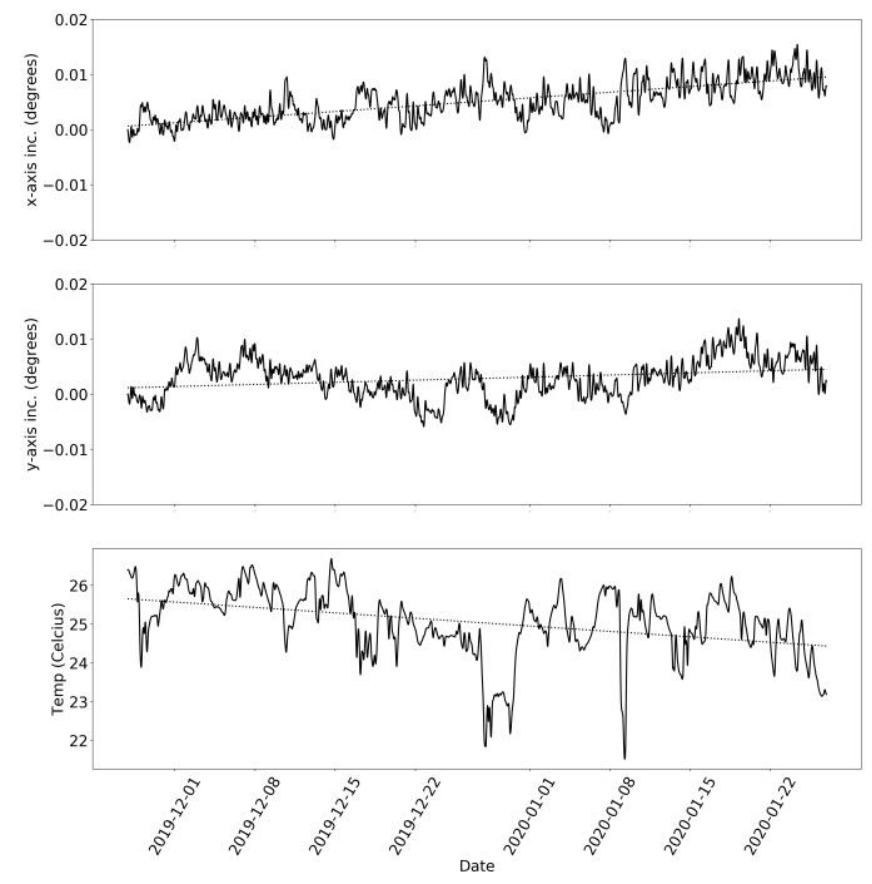

Fig. 10. Long term inclinometer and temperature data records of building. Continuous and dotted lines indicates raw data and linear fitted curve, respectively.

Table III shows the fitted parameters respect to $t$ where;

$$
f(t)=a t+b
$$

equals time dependent functions of raw and compensated inclinometer, and temperature data. As given at Table III, change rate of inclination reduced for $\mathrm{x}$-axis and increased in opposite direction for $\mathrm{y}$-axis after temperature compensation process. Decision of inclination state of building requires longer data acquisition. 
TABLE III

PSEUDO-STATIC MODEL PARAMETERS

\begin{tabular}{lcccl}
\hline \hline \multicolumn{1}{c}{ Data } & $a$ & Unit & $b$ & Unit \\
\hline ix-raw & $+1.689 \mathrm{E}-09$ & $\% / s$ & $+0.651 \mathrm{E}-03$ & ${ }^{o}$ \\
ix-compensated & $+0.970 \mathrm{E}-09$ & $\% / s$ & $+3.105 \mathrm{E}-03$ & ${ }^{o}$ \\
iy-raw & $+0.635 \mathrm{E}-09$ & $\% / s$ & $+1.179 \mathrm{E}-03$ & ${ }^{o}$ \\
iy-compensated & $-0.729 \mathrm{E}-09$ & $\% / s$ & $-2.443 \mathrm{E}-03$ & ${ }^{o}$ \\
Temperature & $-2.312 \mathrm{E}-07$ & ${ }^{o} \mathrm{C} / \mathrm{s}$ & $+2.565 \mathrm{E}+01$ & ${ }^{o} \mathrm{C}$
\end{tabular}

\section{CONCLUSION}

A low-cost system to remotely monitor long-term inclination of a building is successfully realized and tested. Temperature dependency of MEMS based inclinometers should be carefully analyzed before long term data acquisition. Measurement system which includes temperature dependent semiconductor devices should be isolated from temperature variations as much as possible. SCA100T MEMS chip is suitable for structural health monitoring applications, however, resolution of its internal $\mathrm{ADC}$ is insufficient for precise measurement. Using an external 24-bit ADC, resolution of inclination measurement is increased to 0.015372 arc sec., and noise level is $0.002^{\circ}$, approximately. When using the external ADC, the analog outputs of the inclinometer sensor must be filtered with an anti-aliasing first order RC filter, but even this may not suppress noise from free vibrations. Symmetrical PCB geometry should be used to maintain equal dilation and prevent self-bending resulting from temperature expansion. FTP is an efficient way for transferring large data to a remote server especially fire-walled or restricted connections like UDP protocol on EDUROAM network. Wired connection should be used to maintain uninterrupted SHM sensor connections.

\section{ACKNOWLEDGMENT}

This study was funded by Scientific Research Projects Coordination Unit of Istanbul University-Cerrahpasa. Project number: FBA-2017-24225.

\section{REFERENCES}

J. Cai, G. Bu, C. Yang, Q. Chen, and Z. Zuo, "Calculation methods for inter-story drifts of building structures," Adv. Struct. Eng., vol. 17, no. 5, 2014, doi: 10.1260/1369-4332.17.5.735.

[2] G. Artese, M. Perrelli, S. Artese, S. Meduri, and N. Brogno, "POIS, a low cost tilt and position sensor: Design and first tests," Sensors (Switzerland), vol. 15, no. 5, 2015, doi: 10.3390/s150510806.

[3] M. Labibi and M. Mehran, "Novel liquid-based linear capacitive inclination micro-sensor with totally $360^{\circ}$ dynamic range," IET Circuits, Devices Syst., vol. 13, no. 5, 2019, doi: 10.1049/ietcds.2018.5007.

[4] Y. Zhuang, Y. Chen, C. Zhu, R. E. Gerald, Y. Tang, and J. Huang, "A High-Resolution 2-D Fiber Optic Inclinometer for Structural Health Monitoring Applications," IEEE Trans. Instrum. Meas., vol. 69, no. 9, 2020, doi: 10.1109/TIM.2020.2972171.

[5] B. Andò, S. Baglio, V. Marletta, and A. Pistorio, "A Magnetic Fluid-Based Inclinometer Embedding an Optical Readout Strategy: Modeling and Characterization," IEEE Trans. Instrum. Meas., vol. 69, no. 8, 2020, doi: 10.1109/TIM.2020.2964937.

[6] M. Han et al., "Sensitivity improvement of a thermal convection- based tilt sensor using carbon nanotube," Jpn. J. Appl. Phys., vol. 56, no. 6S1, p. 06GF05, Jun. 2017, doi: 10.7567/JJAP.56.06GF05.

D. W. Ha, H. S. Park, S. W. Choi, and Y. Kim, "A wireless MEMSbased inclinometer sensor node for structural health monitoring," Sensors (Switzerland), vol. 13, no. 12, 2013, doi: $10.3390 / \mathrm{s} 131216090$.

[8] H. Huang, R. Xu, and W. Zhang, "Comparative Performance Test of an Inclinometer Wireless Smart Sensor Prototype for Subway Tunnel," Int. J. Archit. Eng. Constr., 2013, doi: 10.7492/ijaec.2013.003.

[9] S. Zhao, Y. Li, E. Zhang, P. Huang, and H. Wei, "Note: Differential amplified high-resolution tilt angle measurement system," Rev. Sci. Instrum., vol. 85, no. 9, 2014, doi: 10.1063/1.4894527.

[10] S. Hou, C. Zeng, H. Zhang, and J. Ou, "Monitoring interstory drift in buildings under seismic loading using MEMS inclinometers," Constr. Build. Mater., vol. 185, 2018, doi: 10.1016/j.conbuildmat.2018.07.087.

[11] B. Andò, S. Baglio, and A. Pistorio, "A low cost multi-sensor system for investigating the structural response of buildings," Ann. Geophys., vol. 61, no. 2, 2018, doi: 10.4401/ag-7702.

[12] B. Akpinar, K. Gürkan, A. A. Dindar, N. O. Aykut, and E. Gülal, "Development of national measurement system for structural health monitoring," in International Congress on Advances in Civil Engineering (ACE 2016), 2016, pp. 1-4.

[13] A. A. Dindar, B. Akpinar, K. Gürkan, E. Gülal, and N. O. Aykut, "Development of low-cost hybrid measurement system," 2018.

[14] S. Martino, L. Lenti, and C. Bourdeau, "Composite mechanism of the Büyükçekmece (Turkey) landslide as conditioning factor for earthquake-induced mobility," Geomorphology, vol. 308, 2018, doi: 10.1016/j.geomorph.2018.01.028.

\section{BIOGRAPHIES}

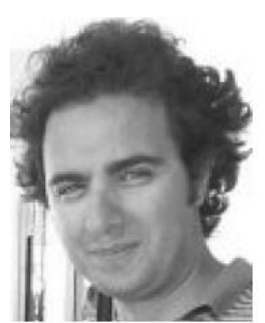

KORAY GURKAN Trabzon, in 1982. He received the B.S. in electrical and electronics engineering in 1999, M.S. and the Ph.D. degrees in Biomedical Engineering from Istanbul University, in 2006 and 2012, respectively.

Since 2016, he has been an Assistant Professor with the Electrical and Electronics Engineering Department, Istanbul University-Cerrahpasa. His research interests include metrology, electronic circuit design and realization, sensor interface, and structural health monitoring. He has been a co-founder of two start-up companies and chairs the scientific advisory board of several $R \& D$ companies.

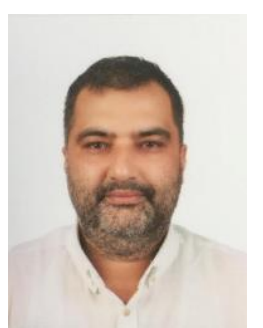

BURAK AKPINAR Canakkale, in 1978. He received the B.S. in geomatic engineering from Yildiz Technical University, Istanbul, in 2000, M.S. and the $\mathrm{Ph} . \mathrm{D}$. degrees in geomatic engineering from Yildiz Technical University, in 2003 and 2009, respectively.

Since 2016, he has been an Associate Professor with the Geomatic Engineering Department, Yildiz Technical University. His research interests include engineering surveying, deformation measurements in engineering structures, Global Navigation and Satellite System (GNSS), Unmanned Aerial Vehicle (UAV)

systems.

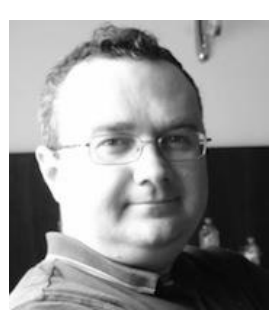

AHMET ANIL DINDAR Erzurum, in 1977. He received the B.S. in civil engineering from Yildiz Technical University, Istanbul, in 1999, M.S. degrees in structural engineering from Istanbul Technical University, Istanbul, in 2002 and the Ph.D. degree in civil engineering from Bogazici University, Istanbul, in 2009.

From 2001 to 2016 , he was a faculty member in Istanbul Kultur University. Since 2016, he has been an Assistant Professor with the Civil Engineering Department, Gebze Technical University, Kocaeli. His research interests include performance based design and structural health monitoring. 